\title{
A Runtime Model Approach for Data Geo-location Checks of Cloud Services
}

\author{
Eric Schmieders, Andreas Metzger, and Klaus Pohl \\ paluno (The Ruhr Institute for Software Technology) \\ University of Duisburg-Essen, Essen, Germany \\ \{eric.schmieders, andreas.metzger, klaus . pohl\}@paluno . uni-due.de
}

\begin{abstract}
Organizations have to comply with geo-location policies that prescribe geographical locations at which personal data may be stored or processed. When using cloud services, checking data geo-location policies during design-time is no longer possible - data geo-location policies need to be checked during run-time. Cloud elasticity mechanisms dynamically replicate and migrate virtual machines and services among data centers, thereby affecting the geo-location of data. Due to the dynamic nature of such replications and migrations, the actual, concrete changes to the deployment of cloud services and thus to the data geolocations are not known. We propose a policy checking approach utilizing runtime models that reflect the deployment and interaction structure of cloud services and components. By expressing privacy policy checks as an st-connectivity problem, potential data transfers that violate the geolocation policies can be rapidly determined. We experimentally evaluate our approach with respect to applicability and performance using an SOA-version of the CoCoME case study.
\end{abstract}

Keywords: Privacy, Cloud Service Management, Service Governance, Runtime Checking.

\section{Introduction}

Privacy regulations such as the EU Data Protection Directive 1 constrain the geographical location of personal data. Therefore, organizations have to comply with data geo-location policies when storing or processing personal data. For instance, the EU Data Protection Directive permits organizations to transfer personal data within the EU only or to such non-EU countries that guarantee sufficient data protection mechanisms.

In case personal data is stored or processed using cloud services, compliance with geo-location policies cannot be checked during design-time, it has to be continuously monitored and checked at runtime. Cloud infrastructures apply replication as well as migration to databases and data processing services in order to scale resources on demand and to accomplish performance, availability, and

${ }^{1}$ http://eur-lex.europa.eu/

X. Franch et al. (Eds.): ICSOC 2014, LNCS 8831, pp. 306-320 2014.

(C) Springer-Verlag Berlin Heidelberg 2014 
cost goals [5,23. These elasticity mechanisms replicate and migrate virtual machines and services among data centers, which may lead to dynamic re-locations of personal data. During design-time, changes to service deployments and consequently to geo-locations of data are unknown and have to be checked during runtime. For instance, two interacting services process personal data and are initially deployed on cloud data centers within the EU. For performance reasons, one service is migrated to a cloud data center located outside the EU. When both services interact after the migration, they exchange personal data across the EU-borders and thus violate data geo-location policies.

Privacy checking approaches such as host geo-location [8, 13] consider the cloud as a black box. These approaches are agnostic to migrations and replications that may occur behind service interfaces. Approaches on access control mechanisms [7, 17 neither consider changes of data geo-locations imposed by migration or replication nor transitive data transfers. In summary, existing approaches are limited in detecting privacy violations that arise from the combination of cloud elasticity and service interactions.

In this paper, we systematically analyze cloud service elasticity in combination with service interactions towards potential policy violations. We propose a novel policy checking approach based on runtime models that covers the identified cases of policy violations. The proposed runtime models reflect the deployment and interaction of cloud services and components. The models are updated when migrations or replications are applied to the reflected cloud applications. By expressing the privacy policy checks as an st-connectivity problem on the runtime models, potential data transfers that violate the geo-location policies can be rapidly determined. The empirical evaluation indicates that the approach is both effective and performant.

The remainder of the paper is structured as follows. Sec. 2 systematically analyzes the changes in the cloud infrastructure that need to be considered to identify data geo-location violations. Sec. 3 discusses the related work. Sec. 4 introduces our policy checking approach. In Sec. 5 we evaluate our approach concerning its effectiveness and performance using the CoCoMe case study. Sec. 6. concludes the paper and provides an outlook to future work.

\section{Cloud Changes impacting on Data Geo-location Policies}

In this section, we systematically analyze the changes in cloud infrastructures that need to be covered in order to detect data geo-location violations. In order to illustrate the different changes, we use the CoCoMe case study.

\subsection{CoCoME Case Study}

The CoCoME case study [18] describes a typical trading application run by a supermarket chain. CoCoME has been used in various empirical evaluations of, e.g., performance predictions and model transformations approaches2. We

\footnotetext{
2 http://sourceforge.net/apps/trac/cocome/
} 
employ a variant of CoCoME that has been adapted to the cloud (within a working group of the DFG Priority Programme "Design For Future" 3 described in, e.g., 14 ). This variant collects shopping transactions of customers in order to offer payback discounts, thereby involving the storage and processing of personal data. In the following, we describe the CoCoMe case study in terms of service and component interactions, the characterization of data, and the definition of data geo-location policies.

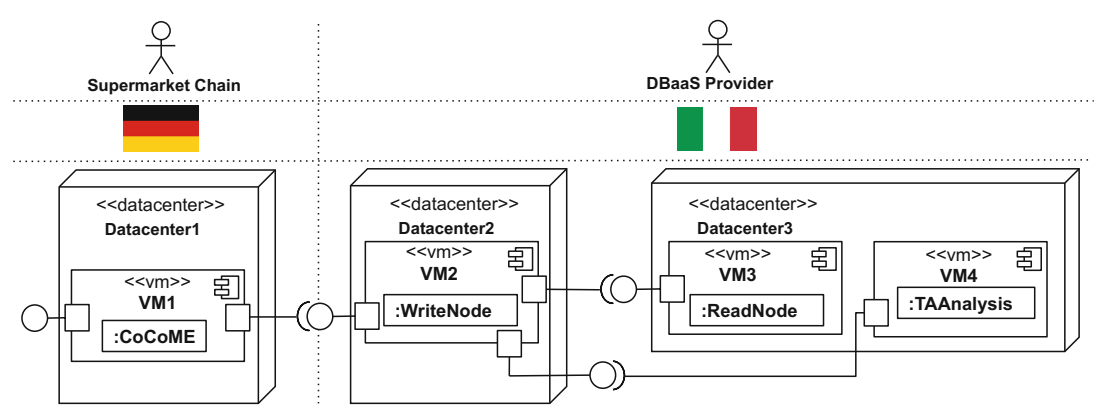

Fig. 1. Initial deployment of the CoCoME application

:CoCoME is the core component of the trading application. It is deployed on virtual machine VM1. :CoCoME provides its features as a service (via an interface) to a web shop as well as to the local stores of the supermarket chain. The trading application uses a data-base as a service $(D B a a S)$ provided by a cloud provider located in Italy. The database is vertically fragmented and distributed among a write node and a read node (further read nodes may be added on demand). The :WriteNode stores personal sales data and the :ReadNode stores anonymized sales data. VM4 executes the transaction analysis component :TAAnalysis. :TAAnalysis accesses the WriteNode and collects all log files of the connected data bases for logging and analysis reasons. Due to the EUDPD, the supermarket chain decides that neither personal identifiable data nor anonymized data (as it may be de-anonymized) shall leave the EU and specifies a corresponding set of policies.

The initial deployment of the virtual machines is shown in Fig. 1. In the following subsection, we use the case study to illustrate situations in which the deployment is changed by replication and migration. The changes lead to violations of data geo-location policies.

\subsection{Data Re-location in the Cloud}

A virtual machine stores data, receives data from other virtual machines, or transfers data to them. Further, a virtual machine can be migrated or replicated.

\footnotetext{
3 http://www.dfg-spp1593.de
} 
Based on these characteristics, cloud elasticity influences data geo-locations (i) when a virtual machine stores personal data and this virtual machine is migrated or replicated to a new geo-location (case 1) and (ii) when data is transferred to a virtual machine by service interactions after migrating or replicating the virtual machine to a new geo-location. Data may be transferred to the new location by directly interacting components and services (case 2) or by indirectly interacting components and services (case 3).

In the following, we examine these three cases that have to be covered to detect potential violations. This allows us to systematically identify the information required to perform geo-location checks at runtime (labelled $R 1$ to $R 8$ ):

\section{- Case 1: A virtual machine that stores personal data is migrated or replicated across data centers.}

In this case, personal data is transferred inside a virtual machine to a remote location, which may violate data geo-location policies. In our case study, $V M 3$ is migrated to Datacenter4 located in the US. The migration of the data base that contains personal data violates the imposed data geo-location policy for personal sales data. Information required for detecting this case: information on components storing data $(R 2)$ of certain types $(R 3)$, information on component deployments to physical resources $\left(R_{4}\right)$, and information on the geo-location information of physical resources $(R 5)$.

- Case 2: A component $A$ stores personal data and interacts with a component $B$. The virtual machine executing $B$ is migrated to a remote data center.

Personal data might be transferred to the remote location, after $B$ has been migrated or replicated. Both cases 2 and 3 allow the derivation of data transfers, which potentially violate geo-location policies. In our case study, $V M_{4}$ is migrated to a data center located in the US. Personal sales data stored at VM2 will be transferred to the :TAAnalysis component located in the US after the migration. The transfer of personal data to the US violates the data geo-location policy. Information required for detecting this case: information $R 2-5$ is required as in case 1 . Furthermore, information about the interaction of the two components is required $(R 1)$.

- Case 3: A component $A$ stores personal data and interacts transitively with a component $C$. The virtual machine executing $C$ is replicated or migrated to a remote data center.

Components may transitively interact among several intermediate components offered by different providers. Once personal data is transferred to a third-party service this service may distribute the data to providers not being aware of the policies applicable to the data. In our case study, VM4 is transferred to a remote data center located in Ireland (other than case 2). VM4 executes the :TAAnalysis component, which logs personal data accessed from VM2. The data center is connected to a back up service from a third party (such as Glacier 4 ). The service distributes backup data (including

4 https://aws . amazon.com/glacier/ 
the personal data) among data centers outside the EU, which violates geolocation policies. Information required for detecting this case: information $R 1-5$ is required as in case 2. Furthermore, explicit or implicit information about transitive data transfers among components is required $(R 6)$.

Checks covering the three cases have to access the relevant information summarized in Tab. 1 .

Table 1. Required information

\begin{tabular}{l|l}
\hline$\#$ & Required information to carry out runtime checks \\
\hline$R 1$ & Interactions of two components \\
$R 2$ & Access of components to locally stored files \\
$R 3$ & Meta-information of stored or processed data \\
$R 4$ & Information on component deployments on physical resources \\
$R 5$ & Geo-location information of physical resources \\
$R 6$ & Explicit or implicit information on transitive data transfers \\
\hline
\end{tabular}

\section{Related Work}

In the related work, we analyze whether existing privacy checking approaches cover the three data re-location cases. Furthermore, we examine if current runtime models provide the required information summarized in Tab. 1 .

\subsection{Privacy Checks during Runtime}

Three major directions of checking geo-location policies during runtime have been investigated in the literature: geo-locating data centers, employing access control mechanisms, and enforcing elasticity rules. Approaches for checking data geo-locations based on service interface locations have been proposed in [8 13,19. Round-trip times of pings sent to the service interfaces are correlated to geographical information in order to determine the geo-location of data centers. This allows to determine, whether service interfaces reside at specific geo-locations. However, the software components behind the service interfaces might be migrated or replicated, while the service interface remains at the same geo-location. For instance, Hadoop data nodes might be replicated to different locations while the request handling node is not migrated. Thus, the concept of using round-trip times of service endpoints to determine data geo-location is not able to cover the cases 1-3 (Sec. 2.2).

Approaches on access control mechanisms [7, 17] equip cloud services with mechanisms that permit or grant data access after matching the client characteristics with the data policies. However, access control mechanisms do not consider changes of data geo-locations imposed by migration or replication of 
the service storing the data (case 1). Moreover, data transfers between the client services and further services are not covered. Transitive data transfers (case 3) that may lead to policy violations thus remain undetected.

Rules for controlling cloud elasticity have been proposed in 21] as well as in the MODACloud 5 and Optimis 6 projects. Those elasticity rules are defined during design time. They are utilized to achieve quality goals, such as response time, energy consumption, cost, and reliability during runtime. However, rules that implement data geo-location policies have to be defined considering the data stored by a virtual machine (case 1) as well as the data, which may be transferred to it (case 2 and 3 ). Yet, this information is not available during design time (see Sec. 2.2), and thus defining geo-location rules during design-time is not feasible.

To summarize, none of the existing approaches cover the cases 1-3. The approaches in [7, 17] cover case 2, but fall short in detecting policy violations resulting from transitive data accesses and cloud elasticity.

\subsection{Runtime Models}

Work on runtime models utilizes sequence-models [15], workflow models [12,20, Markov-chains [6], and state machines [1]. These behavioral models include activities, interactions, and states of reflected applications. Concerning the required information described in Sec. 2 the utilized runtime models lack information on data access $(R 2)$, meta-information of data $(R 3)$, deployment of components to physical resources $\left(R_{4}\right)$, and the geo-location of physical resources $(R 5)$.

Architectural runtime models, such as proposed in [2, 10, 11, 16, combine behavioral aspects of the system with structural information. The utilized runtime models reflect components, workflows executed within the components, and the deployment of components to physical resources. Concerning the required information, the proposed models lack information on data access $(R 2)$, metainformation of data $(R 3)$, and the geo-location of physical resources $(R 5)$.

To summarize, current runtime models do not provide the required information $R 1-6$ to run checks covering cases 1-3.

\section{Runtime Model-Based Policy Checks}

In order to address the limitations of current privacy checking approaches, our approach utilizes runtime models that reflect the deployment and communication structure of cloud services and components. A data geo-location policy $p$ codifies which types of data are forbidden to be stored or processed at specified geographical locations. During runtime, the runtime model $G$ is updated in case of cloud migration or replication automatically. In turn, such model update triggers a check of the model against the data geo-location policies, i.e. $G \models p$.

Below we describe the concepts (meta-model) of the runtime models (Sec.4.1), the formalization of geo-location policies (Sec. 4.2), and the implementation of

\footnotetext{
5 http://www.modaclouds.eu

6 http://www.optimis-project.eu
} 
the policy checks as an st-connectivity problem, and discuss how this covers cases 1-3 (Sec. 4.3).

\subsection{Runtime Model}

The concepts for the runtime models underlying our approach are shown in Fig. 2. The concepts Datacenter, VM, Component, and the relations between them provide the information on components and their deployments required to run the policy check (see $R_{4}$ in Sec. 2). One GeoLocation references several Datacenter $(R 5)$. Modeling this relation is important to facilitate the runtime check (to be discussed in Sec. 4.3). The Component concept subsumes both traditional components and services. Components execute processes (Process) that may interact across components $(R 1)$ and data centers. The meta-model allows defining components that access data through further components and can represent direct or transitive data transfers $(R 6)$. From this relation potential data transfers are derived.

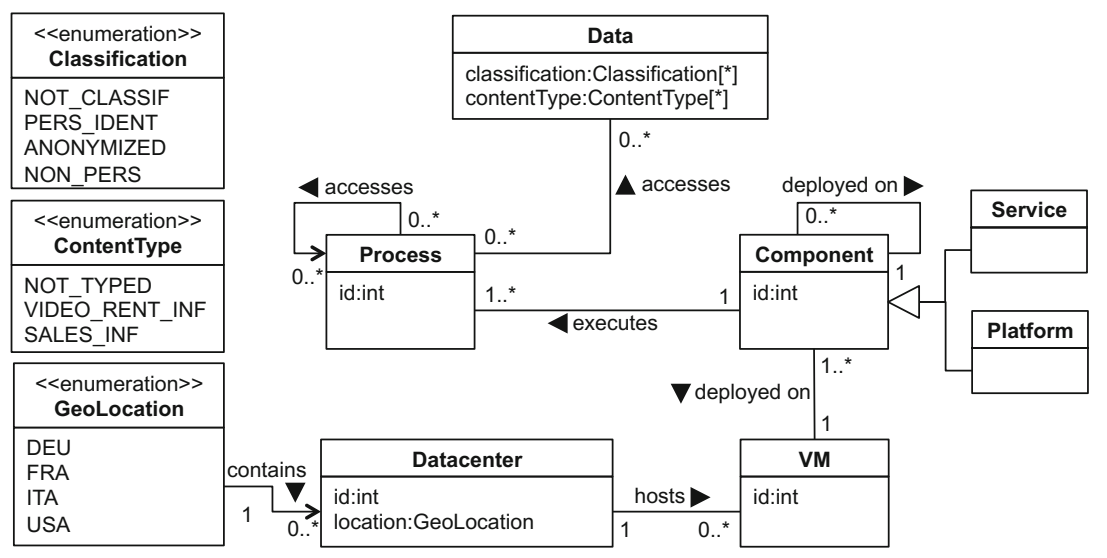

Fig. 2. Relevant concepts of the runtime model

Processes access data that is stored in the component executing the process (R2). Anonymized data can potentially be de-anonymized [24]. Furthermore, accidental or intentional disclosure of different content types is attached to diverse severities and penalties as stipulated, e.g., in the Video Privacy Protection Act7 and the Health Insurance Portability and Accountability Act8. Consequently, data may be treated differently with respect to its classification and content. To support a flexible definition, we enrich the modeled Data entity with a Classification and a ContentType (R3).

\footnotetext{
7 http://www. law. cornell.edu/

8 http://www.cms.gov/
} 
The runtime model may be created manually during the software design phase or may be generated from software artifacts (source code, deployment descriptors etc.) as part of a model-driven engineering process. During runtime, whenever replication or migration changes the deployment or composition of the reflected application, the model has to be updated. Due to space limitations we focus on the presentation and evaluation of the policy checking approach in this paper and examine the monitoring-driven update of the runtime model structure in our future work. However, a comprehensive survey on updating runtime models based on monitoring data can be found in [22].

\subsection{Data Geo-location Policy}

In approaches such as [8] data geo-location policies are defined in natural language. However, we need a formal specification of data geo-location policies in order to run automized checks. To this end, we define a data geo-location policy $p \in P$ as triple $p=(S, T, L)$, with data classifications $S$, data content types $T$, and geo-locations $L$ (in correspondence to classifications, content types, and geo-locations specified in the runtime model). A policy $p$ specifies that every combination $S \times T \times L$ is forbidden. Using sets $S, T$, and $L$ rather than single elements is a design decision helping to reduce the amount of policies to be specified.

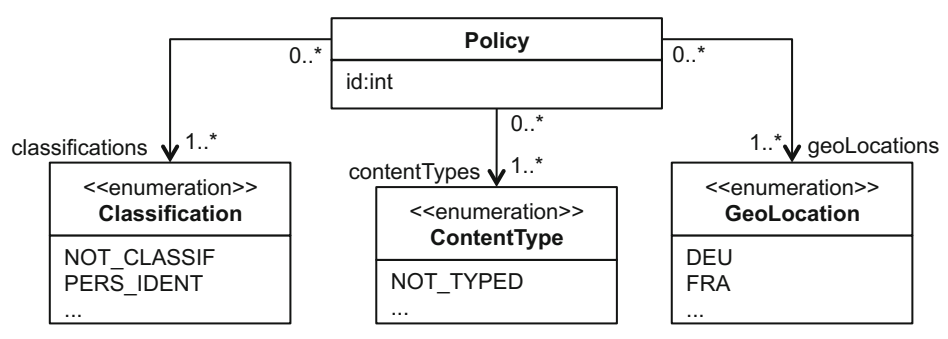

Fig. 3. Relevant concepts for data geo-location policies

In order to implement the geo-location policy concept, existing technologies and languages may be used. The elasticity rules presented in 21] specify the exchange of resources but do not reflect data classifications and content types. Approaches for specifying data access, e.g., work based on XACML9 and [7], do not provide entities for specifying data types and geo-location constraints. However, more generic languages such as WS-Policy 10 or WS-Agreement 11 do not provide dedicated expressions to specify data geo-location constraints but may be extended to formalize the policy concepts described above, such as in [9].

\footnotetext{
9 http://docs.oasis-open.org/xacml/

10 http://www.w3.org/TR/ws-policy/

11 http://www.ogf.org/documents/GFD.107.pdf
} 


\subsection{Policy Check}

The approach specifies the geo-location policy check as an st-connectivity problem on the runtime model. Solving the st-connectivity problem answers the question whether a target node is reachable from a source node in a graph.

To check the reachability, the algorithm selects a subset of geo-location nodes from the runtime model specified in the policy to be checked. The same is performed for the data nodes. After defining both subsets the algorithm checks if there is any path from the subset of geo-locations to the subset of data nodes in the runtime model. If a path exists, it indicates that a potential data transfer violates the checked policy (as defined by the access semantics of the model).

As an example, Fig. 4 shows a simplified version of the runtime model reflecting case 2 (VM4 has been migrated to the US, see Sec. 2.1). There exists a path with geoLocation(usa) as start node $v_{s}$ and Data(1, [PERS_IDENT], $\left[H E A L T H_{-} I N F\right]$ ) as target node $v_{t}$, i.e. personal data may flow into the US. The existence of this path indicates the violation of the policy described in the case study ("storing or processing personal data in the USA is forbidden").

The algorithm of the policy check is shown in Alg. 1.

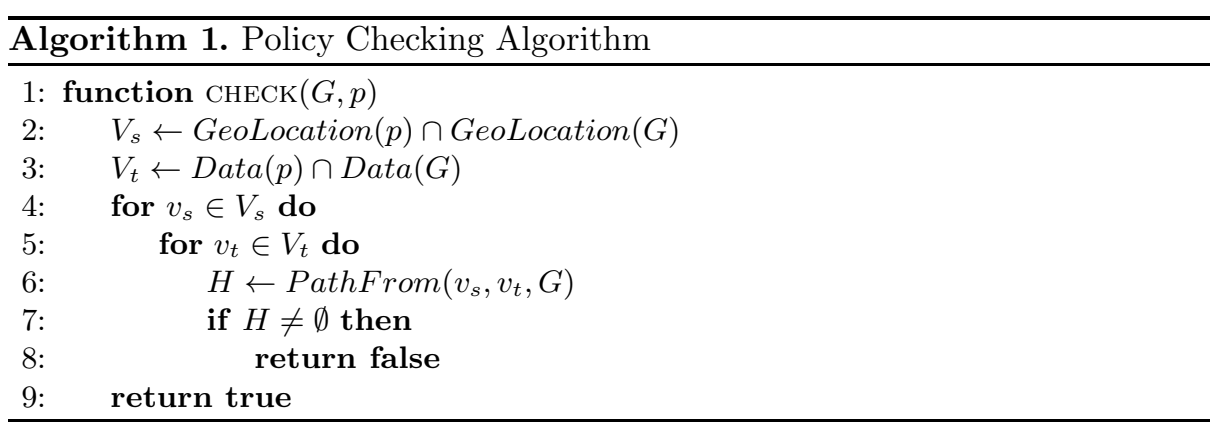

We choose st-connectivity over generic model checking approaches because st-connectivity is sufficient to solve the checking problem. Due to their generality, model checkers have a wide scope of application but also may impose high performance needs. Thus, runtime checking approaches (such as [3, 12, 20]) typically propose tailored concepts for solving specific problems. Furthermore, the st-connectivity problem is NL-complete and thus solvable within polynomial time (analyzed in Sec. 5). In the following, we describe the foundation of the geo-location check.

Definition 1. Let $G$ be a directed graph $G=(V, E)$ (as depicted in the example in Fig. (4). Vertices $V$ are the entities of the runtime model. Edges $E$ are the relations of the runtime model. Let policy $p$ be defined as triple $p=(S, T, L)$ with data classifications $S$, content types $T$, and geo-locations $L$. 


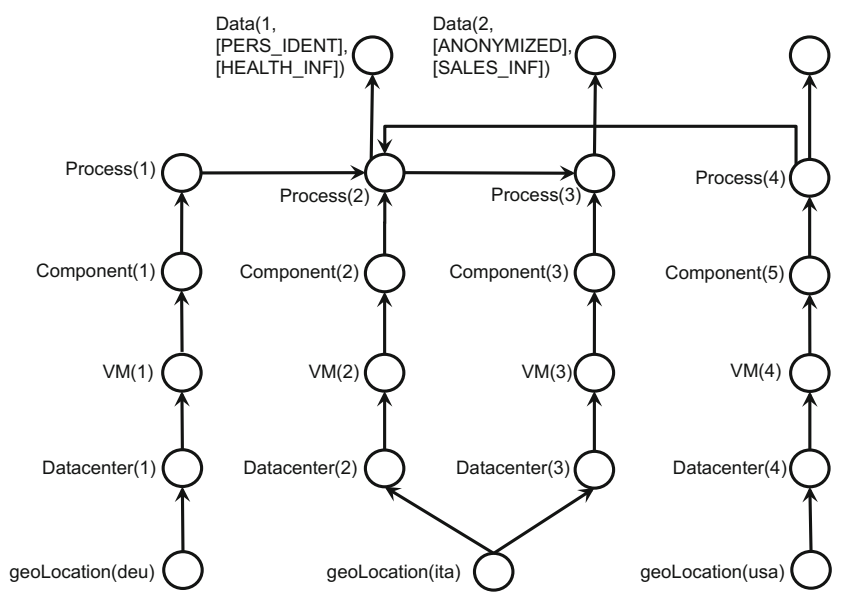

Fig. 4. Runtime model instance of case 2

Definition 2. Let $V_{s}$ be the intersection from geo-locations in $G$ and $p$ with $V_{s}=\{v \mid v \in V \wedge$ GeoLocation $(v) \in L\}$ serving as start nodes. Let $V_{t}$ be the subset of data nodes from $G$ specified in $p$ with $V_{t}=\{v \mid v \in V \wedge$ Classifcation $(v) \in$ $S \wedge$ ContentType $(v) \in T\}$. Let $H_{v_{s}, v_{t}}=\left(v_{s}, \ldots, v_{t}\right)$ be a path in $G$ with $v_{s} \in V_{s}$ and $v_{t} \in V_{t}$.

With Def. 1 we define the runtime model as graph $G$ and with Def. 2 we define a path $H$ in $G$. When searching path $H$ in graph $G$, modeling the relation between GeoLocation and Datacenter is important. Based on this, the runtime model can be transversed from GeoLocation entities $V_{s}$ to data nodes $V_{t}$, which allows defining the policy check as st-connectivity problem with Def. 3 .

Definition 3. Given a graph $G$ and a policy $p$, we define the model check $G \mid p$ as function $f(G, p): G \times\{p\} \rightarrow\{$ true, false $\}$

$$
f(G, p)=\left\{\begin{array}{rll}
\text { true } & : & \neg\left(\exists v_{s} \exists v_{t}: H_{v_{s}, v_{t}}, \text { with } v_{s} \in V_{t} \wedge v_{t} \in V_{t}\right) \\
\text { false } & : & \text { else }
\end{array}\right.
$$

where "true" means the checked equation holds and "false" means the policy is violated. 2.

The checking approach covers the data re-location cases 1-3 introduced in Sec.

- Case 1 is covered when both $v_{t}$ and the host where the data reside are at geo-location $v_{s}$.

- Case 2 is covered when a component executed at geo-location $v_{s}$ directly accesses $v_{t}$ from a remote component.

- Case 3 is covered when a component at geo-location $v_{s}$ accesses $v_{t}$ from a remote component transitively through further components. 
The implementation of the runtime check may base on algorithms for graph traversal. For instance, basic breadth-first search or depth-first search may be applied as well as optimized variants such as the $A^{*}$ algorithm.

\section{$5 \quad$ Experimental Evaluation}

The experimental evaluation aims for analyzing the effectiveness and performance of the geo-location policy checking approach. Here, effectiveness refers to the capability of identifying potential data transfers that may violate data geo-location constraints. Performance refers to the time consumed for checking the violations and indicates how timely one may be informed about violations.

The set up of the experiments is based on the combination of a simulated cloud environment and the prototypical implementation of our approach. The set up includes a runtime model, a set of data geo-location policies, a prototypical implementation of the runtime checking approach using depth first search, and a simulator that simulates replication and migration of virtual machines. We implemented the runtime and policy meta models as Ecore instances 12 . The runtime model reflects the SOA-version of CoCoME (see Sec. 2.1) and includes 22 data centers distributed among five countries, four virtual machines, seven components, and six processes accessing two different types of personal data. The simulation environment allows us to run controlled, reproducible functional tests and to examine policy checking performance without provider limitations or side effects.

\subsection{Experiment on Effectiveness}

To determine the effectiveness of our approach, we investigate "whether the proposed approach correctly identifies potential data transfers that may violate data geo-location constraints?" To evaluate the expressiveness, we modeled the functionalities of two CoCoME use cases (use case 1 and 7 from the CoCoMEspecification 13 ). In use case 1 , a web shop customer adds products to the shopping cart. In use case 7 , the sales manager changes the price of a product. Use case 1 serves as a positive example that includes potential policy violations. Use case 7 serves as a counterexample as it does not include any policy violation. We create six equivalence partitions to test the effectiveness of the checking approach systematically. The six partitions result from defining positive and negative tests for each of the three data re-location cases (see Sec. 2.2). For each partition, we define up to three test cases in which we provide the policy checker with input that corresponds to the specified equivalence partition. For instance, a valid positive test case of data re-location case 3 includes (i) a runtime model that reflects the transitive transfer of anonymized sales data into the USA, (ii) a policy that forbids to store or process anonymized sales data in the USA, and (iii) the detected policy violation as the expected test result.

\footnotetext{
12 http://www.eclipse.org/modeling/emf/

13 http://sourceforge.net/projects/cocome/
} 
Our approach passes the functional tests and thus correctly identifies all three cases of policy violations. Of course, the generalizability (external validity) of these findings is limited by the fact that we examined the expressiveness by means of a single case study. Although CoCoME is used in a multitude of empirical studies, we thus plan to apply our approach to further applications in our future work.

\subsection{Experiment on Performance}

In order to explore the performance, we examine "how the runtime model complexity impacts on the response time of the proposed approach?" To this end, we stepwise increase the complexity of the runtime model (independent variable) and measure the response times of the policy checks (dependent variable).

The complexity of a graph with respect to the time consumption of depth first searches is determined by the numbers of edges within a graph (the runtime model), which we use as a complexity metric. To stepwise increase the complexity of the initial model, we iteratively replicate virtual machines and insert new interactions (reflected in the model). To give an example: the model complexity of 1000 results from the edges of 68 virtual machines and 119 modeled processes. We stop the experiment at the complexity of 135183 , which results from the edges of 814 virtual machines and 1414 processes. We execute the Java-based prototype (SDK 1.6) on a 2,3 GHz Quad-Core i7 machine running OS X 10.

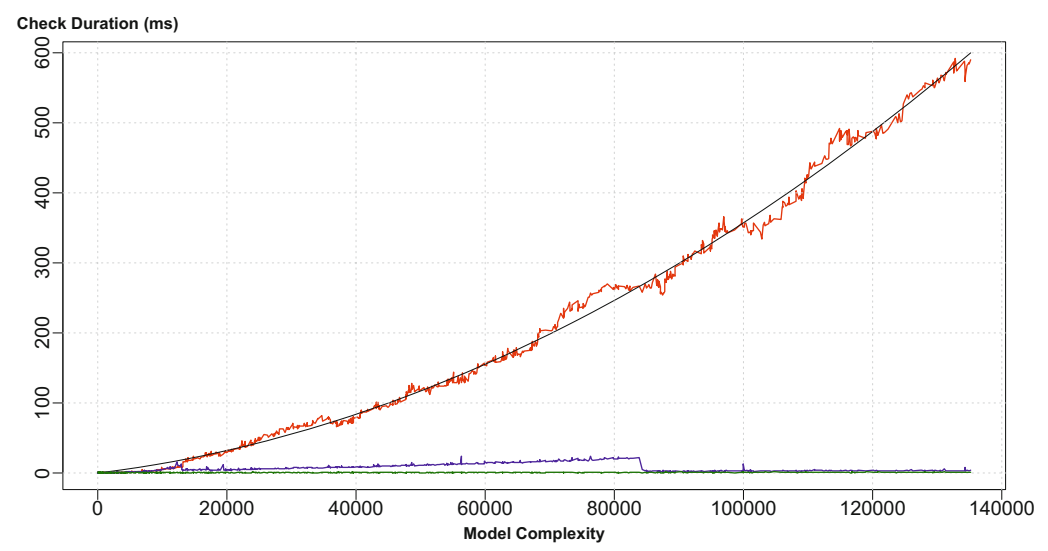

Fig. 5. Measured response times of policy checks

We measure three different situations of runtime policy checking: "best case", "worst case", and "typical case". Fig. 5 shows the measured time consumption (y-axis) over the model complexity (x-axis). We observed the following:

- In the "best case" (green flat graph), personal data is stored at a geo-location and this storage relation is forbidden by one of the policies. The search depth is low and the checks stop at $t_{b} \leq 1 \mathrm{~ms}$. 
- In the "worst case" (red graph) the search algorithm has to traverse every possible path entirely to detect the policy violation. The measured growth in checking durations of worst cases is of polynomial time as the upper bound can be described by a quadratic expression (black curve in the figure), i.e. $t_{w}=1.112 \times 10^{-3} * x+2.460 \times 10^{-8} \times x^{2}$ with $x$ as model complexity (expression estimated by non-linear regression). This indicates that the growth indeed maps to the analytical complexity of the st-connectivity problem.

- The "typical case" experiment is repeated several times with different seed values. We observe that the checking durations increase according to $t_{w}$ (all paths are explored) until a violation occurs. After this, the checking durations increase linear or remain constant. Durations of the "typical case" are within $\left[t_{b}, t_{w}\right]$. Fig. [5] shows one example run (blue bouncing graph). First, the run duration increases according to $t_{w}$ until the complexity of 12275 at which the checked model exhibits a policy violation. After the violation occurs, the number of visited nodes required for detecting the violation grows linear due to replication adding each step up to one node to the search path. At a complexity of 83847 , a service interaction is randomly inserted. The interaction connects a service located at the excluded geo-location to a service that processes personal data. This almost reduces the time checking duration to $t_{b}$. Further runs of the "typical case" show similar behaviors.

An amount of more than 800 virtual machines is realistic for large applications as, for instance, Hadoop-clusters typically utilize several hundreds of data nodes (see [4]). However, our approach is still able to check the worst case for large cloud applications ( $>500$ virtual machines) in less than one second (cf. $\left.t_{b}\right)$. Of course, due to the decision to simulate the cloud, the experimental design may have limitations towards construct validity. There may be further factors influencing the performance of the approach in a productive cloud environment. For instance, delays of monitoring data may result from geographical distances between the monitoring probes and the place where the policy checker resides. These limitations have to be tackled in future experiments conducted on real cloud infrastructures.

\section{Conclusion and Future Work}

We analyzed the problem of checking the compliance of data geo-location policies for cloud services. We identified and described three cases that may violate data geo-location policies. These cases result from changes in service interactions in combination with cloud elasticity. In contrast to existing policy checks, the proposed approach considers direct and indirect data transfers resulting from service interactions. Initial experimental evidence indicates that the proposed approach is able to correctly identify violations and that it may do so - even for large cloud settings - with very fast response times. As a part of future work, we plan to apply our approach to other application types as well. Further, we aim for investigating the approach's effectiveness in realistic cloud environments. 
To this end, we will investigate its violation detection capabilities with respect to precision, recall, and further evaluation metrics. In addition, we will complement our approach by leveraging cloud monitoring data to update the proposed runtime models.

Acknowledgements. This work was partially supported by the DFG (German Research Foundation) under the Priority Programme "SPP1593: Design For Future - Managed Software Evolution" (grant PO 607/3-1).

\section{References}

1. van der Aalst, W., Schonenberg, M., Song, M.: Time prediction based on process mining. Information Systems 36(2) (Apr 2011)

2. Brosig, F., Huber, N., Kounev, S.: Automated extraction of architecture-level performance models of distributed component-based systems. In: 2011 26th IEEE/ACM International Conference on Automated Software Engineering, ASE (2011)

3. Canfora, G., Di Penta, M., Esposito, R., Villani, M.L.: A framework for QoS-aware binding and re-binding of composite web services. Journal of Systems and Software 81(10) (2008)

4. Chen, Y., Alspaugh, S., Katz, R.: Interactive analytical processing in big data systems: A cross-industry study of MapReduce workloads. Proc. VLDB Endow. 5(12) (August 2012)

5. Copil, G., Moldovan, D., Truong, H.-L., Dustdar, S.: Multi-level elasticity control of cloud services. In: Basu, S., Pautasso, C., Zhang, L., Fu, X. (eds.) ICSOC 2013. LNCS, vol. 8274, pp. 429-436. Springer, Heidelberg (2013)

6. Epifani, I., Ghezzi, C., Mirandola, R., Tamburrelli, G.: Model evolution by runtime parameter adaptation. In: 31st Internal Conference on Software Engineering (ICSE) (2009)

7. e-Ghazia, U., Masood, R., Shibli, M.A.: Comparative analysis of access control systems on cloud. In: 2012 13th ACIS International Conference on Software Engineering, Artificial Intelligence, Networking and Parallel Distributed Computing (SNPD) (2012)

8. Gondree, M., Peterson, Z.N.: Geolocation of data in the cloud. In: Proceedings of the Third ACM Conference on Data and Application Security and Privacy, CODASPY 2013. ACM, New York (2013)

9. Gutiérrez, A.M., Cassales Marquezan, C., Resinas, M., Metzger, A., Ruiz-Cortés, A., Pohl, K.: Extending WS-Agreement to support automated conformity check on transport and logistics service agreements. In: Basu, S., Pautasso, C., Zhang, L., Fu, X. (eds.) ICSOC 2013. LNCS, vol. 8274, pp. 567-574. Springer, Heidelberg (2013)

10. van Hoorn, A., Rohr, M., Hasselbring, W.: Engineering and continuously operating self-adaptive software systems: Required design decisions. In: Engels, G., Reussner, R.H., Momm, C., Stefan, S. (eds.) Design for Future 2009, Karlsruhe, Germany (November 2009)

11. Huber, N., Brosig, F., Kounev, S.: Modeling dynamic virtualized resource landscapes. In: Proceedings of the 8th International ACM SIGSOFT Conference on Quality of Software Architectures (2012) 
12. Ivanović, D., Carro, M., Hermenegildo, M.: Constraint-based runtime prediction of sla violations in service orchestrations. In: Kappel, G., Maamar, Z., MotahariNezhad, H.R. (eds.) Service Oriented Computing. LNCS, vol. 7084, pp. 62-76. Springer, Heidelberg (2011)

13. Juels, A., Oprea, A.: New approaches to security and availability for cloud data. Commun. ACM 56(2) (February 2013)

14. Jung, R., Heinrich, R., Schmieders, E.: Model-driven instrumentation with kieker and palladio to forecast dynamic applications. In: Symposium on Software Performance: Joint Kieker/Palladio Days 2013. CEUR (2013)

15. Maoz, S.: Using model-based traces as runtime models. Computer 42(10) (2009)

16. von Massow, R., van Hoorn, A., Hasselbring, W.: Performance simulation of runtime reconfigurable component-based software architectures. In: Crnkovic, I., Gruhn, V., Book, M. (eds.) ECSA 2011. LNCS, vol. 6903, pp. 43-58. Springer, Heidelberg (2011)

17. Park, S., Chung, S.: Privacy-preserving attribute distribution mechanism for access control in a grid. In: 21st International Conference on Tools with Artificial Intelligence (2009)

18. Rausch, A., Reussner, R., Mirandola, R., Plášil, F. (eds.): The Common Component Modeling Example. LNCS, vol. 5153. Springer, Heidelberg (2008)

19. Ries, T., Fusenig, V., Vilbois, C., Engel, T.: Verification of data location in cloud networking. IEEE (December 2011)

20. Schmieders, E., Metzger, A.: Preventing performance violations of service compositions using assumption-based run-time verification. In: Abramowicz, W., Llorente, I.M., Surridge, M., Zisman, A., Vayssière, J. (eds.) ServiceWave 2011. LNCS, vol. 6994, pp. 194-205. Springer, Heidelberg (2011)

21. Suleiman, B., Venugopal, S.: Modeling performance of elasticity rules for cloudbased applications. In: 2013 17th IEEE International Enterprise Distributed Object Computing Conference (EDOC) (September 2013)

22. Szvetits, M., Zdun, U.: Systematic literature review of the objectives, techniques, kinds, and architectures of models at runtime. Software \& Systems Modeling (December 2013)

23. Vaquero, L.M., Rodero-Merino, L., Buyya, R.: Dynamically scaling applications in the cloud. ACM SIGCOMM Computer Communication Review 41(1) (2011)

24. Zang, H., Bolot, J.: Anonymization of location data does not work: A large-scale measurement study. In: Proceedings of the 17th Annual International Conference on Mobile Computing and Networking. ACM, New York (2011) 